\title{
An inventory of medicinal products causing skin rash: Clinical and regulatory lessons
}

\author{
ROBERT ANCUCEANU ${ }^{1}$, MIHAELA DINU ${ }^{1}$, FLORENTINA FURTUNESCU ${ }^{2}$ and DANIEL BODA ${ }^{3,4}$ \\ ${ }^{1}$ Department of Pharmaceutical Botany and Cell Biology, Faculty of Pharmacy, 'Carol Davila' University of \\ Medicine and Pharmacy, 020956 Bucharest; ${ }^{2}$ Department of Public Health and Management, Faculty of Medicine, \\ 'Carol Davila' University of Medicine and Pharmacy, 050463 Bucharest; ${ }^{3}$ Dermatology Research Laboratory, \\ 'Carol Davila' University of Medicine and Pharmacy, 050474 Bucharest; ${ }^{4}$ Carol Medical Center, 010626 Bucharest, Romania
}

Received September 21, 2018; Accepted May 27, 2019

DOI: $10.3892 /$ etm.2019.7837

\begin{abstract}
A variety of medicinal products have been associated with rash and normally this information should be available in the Summary of Product Characteristics (SmPCs). Our study aimed to investigate the frequency of rash as an adverse drug reaction, based on the information provided by SmPCs of 1,048 single active substances (international non-proprietary names) authorized in the United Kingdom. Data on rash frequency was collected from each SmPC using automated searches based on selected keywords. Data analysis was carried out using R, v. 3.4. We found that over $90 \%$ of the medicines used orally or by injection may be associated with rash as an adverse event, the most common classes being protein kinase inhibitors, anticancer medicinal products, monoclonal antibodies, biologicals, antivirals and retinoids, with high variations in rash frequency for products within the same class, but also for products with the same active substance. Analysis of SmPCs revealed the need to increase
\end{abstract}

Correspondence to: Professor Mihaela Dinu, Department of Pharmaceutical Botany and Cell Biology, Faculty of Pharmacy, 'Carol Davila' University of Medicine and Pharmacy, 6 Traian Vuia Street, Sector 2, 020956 Bucharest, Romania

E-mail: mihaela.dinu@umfcd.ro

Abbreviations: ACE, angiotensin-converting enzyme; ADHD, attention deficit hyperactivity disorder; ADR, adverse drug reaction; CIOMS, Council for International Organizations of Medical Sciences; DMARDs, disease-modifying antirheumatic drugs; DPP4, dipeptidyl peptidase 4; DRESS, drug-reaction with eosinophilia and systemic symptoms; eMC, electronic Medicines Compendium; FDC, fixed-dose combination; GLP1, glucagon-like peptide 1; MAH, marketing authorization holder; MedDRA, Medical Dictionary for Regulatory Activities; NSAID, nonsteroidal anti-inflammatory drug; NMDA, N-methyl-D-aspartate; PDE5, phosphodiesterase type 5; SGLT2, sodium-glucose co-transporter 2; SmPC, Summary of Product Characteristics; TNF, tumor necrosis factor

Key words: rash, summary of product characteristics, regulatory, medicinal products, adverse drug reaction homogeneity in reporting rash frequency, by using Council for International Organizations of Medical Sciences classification, and Medical Dictionary for Regulatory Activities coding in a more standardized manner, and also the need to include more safety endpoints in clinical trials and to use better the safety results for publication and updating the SmPCs.

\section{Introduction}

A 'rash' is defined as a 'sudden skin eruption' and is the main driver of patients to visit a dermatologist. A rash is often the result of an underlying disease (1), but a variety of medicinal products have also been associated with rashes. An eruptive skin rash associated with fever or other systemic symptoms is sometimes described as an 'exanthema' (1) or 'maculopapular rash', although (as shown below), the term (or its derivation, 'exanthematous eruptions') is used to describe the most common form of cutaneous eruption, consisting in an acute and extensive eruption of small fixed erythematous spots that become whitish or pale when applying pressure (2).

It has been estimated that $\sim 1-7 \%$ of all hospital admissions or referrals from primary care are related to skin adverse drug reactions (ADRs) $(3,4)$. Of these, the majority (almost half) are represented by exanthema (maculopapular rash), as confirmed by multiple observational studies (4-6) and it has been claimed that almost all medicines have been associated with rash (7).

Allergic and non-allergic hypersensitivity reactions. Immune-mediated adverse drug reactions, generally described as 'hypersensitivity reactions' or 'allergic reactions/allergy' are not limited to rash, but they may include it, as they may be expressed in a variety of clinical forms, from maculopapular exanthema to urticaria, angioedema or anaphylaxis (8).Equality is often placed between 'hypersensitivity reactions' and 'allergic reactions', nevertheless not all drug hypersensitivity reactions are of an allergic nature. The term 'nonallergic drug hypersensitivity reactions' is used in accordance with the international consensus to describe drug reactions similar to allergy, but for which no immunological mechanism has been proven (9). Instead, the phrase 'drug allergy' is reserved for those cases where an immune mechanism involving either antibodies or $\mathrm{T}$ cells mediates the reaction. The international 
consensus recommendation is that for general communication purposes in the case of a suspicion of drug allergy the term 'drug hypersensitivity reaction' should be used (9). In a cross-sectional study among a general population sample a prevalence of $7.8 \%$ of 'drug allergy' has been found based on self-report by the responders (10) (since this was self-reported, and thus the allergic nature was only suspected, the term 'drug hypersensitivity reaction' would have been preferable).

Immediate and delayed allergic reactions. According to their onset, drug induced allergic reactions may be immediate and delayed (non-immediate). Immediate drug induced allergic reactions occur soon after administration of the causative agent and are likely to be mediated by IgE (9). Although periods as short as $1 \mathrm{~h}$ have been proposed and are still used (11), it has been rightly pointed out that a reasonable period to label one drug reaction as 'immediate' should be longer, up to $6 \mathrm{~h}(9,12)$. Immediate allergic reactions may manifest through a diversity of symptoms: urticaria, angioedema, anaphylaxis or anaphylactic shock, conjunctivitis, rhinitis, bronchospasm or even gastrointestinal symptoms (nausea, vomiting, diarrhoea and abdominal pain) $(9,11,13)$. Delayed drug hypersensitivity reactions usually occur after several or many days of treatment, although occurrences as soon as $1 \mathrm{~h}$ after the initial drug administration are possible (9).

Rash classification. Although drug rashes may be classified according to their mechanism of action, such classification is usually of little use in diagnosing and managing an undifferentiated rash in clinical settings. Therefore, a morphological classification in seven groups has been proposed for the cutaneous rashes most frequently encountered in the clinical practice, the first three being the most common and the last more rare: a) exanthema (maculopapular rash): simple exanthemas (typically maculopapular rash) or complex exanthemas (such as DRESS - drug-reaction with eosinophilia and systemic symptoms); b) dermatitis (eczema): phototoxic reactions, photoallergic reactions, primary dermatitis reactions and drug induced xerosis; c) urticarial reactions: anaphylactic and anaphylactoid reactions, acetylsalicylic acid associated urticarial (chronic urticarial exacerbation by aspirin), angioedema, urticarial vasculitis, serum-sickness-like reaction; d) pustular rash: acute generalized erythematous pustulosis (AGEP) and DRESS; e) blistering rash: fixed-drug eruption, Stevens-Johnson syndrome/toxic epidermal necrolysis (SJS-TEN), drug-induced pemphigus, drug-induced bullous pemphigoid, pseudoporphyria, drug-induced linear IgA bullous dermatosis; f) purpuric rash: drug-induced vasculitis, warfarin-induced skin necrosis, heparin-induced skin necrosis; and g) Erythrodermic rash (2).

Summaries of productcharacteristics. The Summary of Product Characteristics (SmPCs) represent the official information approved by the competent authorities granting marketing authorizations for medicinal products within the European Union, intended for healthcare professionals and allowing them to use a specific product safely and effectively $(14,15)$. Although recognized as a comprehensive source of information (16), in the last 10-15 years, SmPCs have been criticized for a number of shortfalls in their structure (16) and in the information they convey, with respect to clinical pharmacology contents (17), therapeutic drug monitoring $(18,19)$, dose adjustment in renal impairment $(20,21)$, drug-drug $(16,22)$ and drug-food interactions (23), use of medicinal products in pregnancy and lactation (15) or the information provided about biosimilar medicinal products (24).

Although various narrative reviews have been published on rash and other adverse drug reactions in general (2,13,25-28), as well as for different therapeutic groups (29-33), no comprehensive review of rash as an adverse effect of medicines has been published. In the present study we report on our investigation of the frequency of rash as an adverse drug reaction, based on the information provided by SmPC. We also critically reflect on shortfalls identified in the way this information is included in SmPC and the inconsistencies we have identified.

\section{Materials and methods}

SmPCs of medicines corresponding to 1,048 single active substances (international non-proprietary names) authorized in the United Kingdom (irrespective of the authorization procedure) were retrieved from the electronic Medicines Compendium (eMC) website (https://www.medicines.org.uk/ emc). When more than a quarter of the SmPC were retrieved and analysed, the eMC migrated its website to a new version, while still making available the old version of the website. To have consistency, we have kept using the old version of the website throughout the data collection and verification. Only medicinal products containing a single active substance were used, excluding fixed-dose combinations (FDCs) or products containing various mixtures, such as herbal extracts or blood fractions. Salts of different organic cations have been considered the same active substance, but esters have been counted as different [e.g., triamcinolone hexacetonide and triamcinolone acetonide have been counted as different products, because clinical data have indicated that they behave similarly only in certain conditions, e.g., the effect of the latter is equivalent to that of the former if used at a double dose (34)]. Instead, different names for the same ester have been considered as a single active substance and product, respectively (e.g., triptorelin embonate and triptorelin acetate have been considered as two distinct substances, whereas triptorelin embonate and triptorelin pamoate have been considered as the same substance, although they were indexed under different active substance names on the $\mathrm{MC}$ website). If several products were authorized for the same active substance (e.g., generic products) we retrieved two (if only two products were available) or three SmPCs (if more than two products were available). Thus, a total of 1743 of SmPC were retrieved and analysed for the purpose of this study. We have limited our search to those SmPC referring to active substances that are used on an oral or parenteral route of administration, with the exclusion of topical or other routes (e.g., rectal, inhalation). Sublingual tablets were included, whereas transdermal patches, although systemic, were excluded.

Data on rash frequency was collected from each SmPC using automated searches based on the following keywords: 'rash', 'exanthema', 'urticaria', 'hypersensitivity', 'anaphylactoid', 'anaphylactic' (photosensitivity was not 
included). Initially we intended to use exclusively the keyword 'rash', but by comparing different SmPCs for the same active substance we found that sometimes the alternative keywords were used for the same active substance. We have also visually examined section 4.8 ('Undesirable effects'), in the part of tabulated adverse events or for the paragraphs under the headings 'Skin and subcutaneous tissue disorders', 'Immune system disorders', 'General disorders and administration site conditions' or equivalent headings in older SmPCs, to identify other terms that may potentially describe rash. We have discovered during the writing of this study that the SmPCs for a small number of products have become unavailable on the eMC website (e.g., lomitapide, regadenoson and vecuronium).

For each active substance and from every SmPC corresponding to that particular active substance, information on rash frequency was collected, using the conventional language currently used by the SmPC template within European Union: very common $(\geq 1 / 10)$; common $(\geq 1 / 100$ to $<1 / 10)$; uncommon $(\geq 1 / 1,000$ to $<1 / 100)$; rare $(\geq 1 / 10,000$ to $<1 / 1,000)$; very rare $(<1 / 10,000)$. The inconsistencies observed among different SmPCs for the same active substance determined us to use certain conventions in tabulating the frequencies. Rather surprisingly, for the same active substance in a number of cases different SmPC included different frequencies; in such cases the highest frequency was tabulated (but an annotation was made in the collection form). The same solution was adopted when different frequencies were stated inside the same SmPC (either for data referring to different indications, e.g., different frequencies found in clinical trials performed in different indications, or for data referring to two types of rash, e.g., maculopapular rash and DRESS). In SmPCs approved rather long ago (although they may have been relatively recently revised, but have been initially approved longer time ago), words like 'rare' or 'rarely' do not have the quantitative meaning they have in the current SmPCs, therefore if such was the case, the frequency for such SmPCs was tabulated as 'not known'. Where for the same product both 'rash' and 'urticaria' or other terms were listed among adverse events, we tabulated the higher frequency. Rash listed as an injection site reaction was excluded. The standard contraindication 'Hypersensitivity to the active substance or to any of the excipients', which is copy-pasted automatically in any SmPC was not considered a statement about the occurrence or frequency of rash for any product.

Statistical analysis. The data analysis was carried out in the computing and programming environment R, v. 3.4 .4 (35). Proportions were compared using the Fisher's exact test, at a confidence level of 0.995 .

\section{Results}

The distribution of rash frequencies among the 1,048 active substances is shown in Table I.

Rash as a 'very common' adverse reaction. Approximately $10 \%$ of all medicinal products included in the analysis may cause rash in $>10 \%$ of the treated patients [Table SI (https://figshare. com/articles/Untitled_Item/7144277)].

Among these, about one quarter (27 active substances, $23.68 \%$ of the subgroup having rash as a very common
Table I. Distribution of rash frequencies among medicinal products (per active substance) administered by oral or injectable route.

\begin{tabular}{lrr}
\hline Frequency & Number & \multicolumn{1}{c}{$\%$} \\
\hline Very common $(\geq 1 / 10)$ & 114 & 10.89 \\
Common $(\geq 1 / 100$ to $<1 / 10)$ & 267 & 25.50 \\
Uncommon $(\geq 1 / 1,000$ to $<1 / 100)$ & 218 & 20.82 \\
Rare $(\geq 1 / 10,000$ to $<1 / 1,000)$ & 98 & 9.36 \\
Very rare $(<1 / 10,000)_{\text {Not known }}^{\mathrm{a}}$ & 33 & 3.15 \\
None $^{\mathrm{b}}$ & 235 & 22.45 \\
Total & 82 & 7.83 \\
\hline
\end{tabular}

an the up-to-date SmPC, the 'not known' frequency label is used when the frequency cannot be estimated from the current data. In our study, however, the same label has also been used for the older SmPC, in which the CIOMS classification has not been applied (e.g., when the SmPC mentioned rash as an example of adverse reaction occurring in 'isolated cases', or 'rarely', the 'not known' label was used). 'The 'none' label was used for those product/active substances for which rash or an alternative term suggesting rash as a possible adverse reaction (e.g., hypersensitivity, allergy, urticaria) was found in SmPCs.

adverse reaction) belong to the larger class of protein kinase inhibitors (most of them tyrosine kinases, but also a few serine/threonine or mitogen-activated protein kinases): afatinib, alectinib, axitinib, bosutinib, cabozantinib, ceritinib, cobimetinib, crizotinib, dabrafenib, dasatinib, erlotinib, gefitinib, ibrutinib, imatinib, lapatinib, lenvatinib, nilotinib, nintedanib, osimertinib, pazopanib, ponatinib, regorafenib, sorafenib, sunitinib, trametinib, vandetanib, and vemurafenib.

An additional group of 19 active substances (e.g., 16.67\% of those with rash as a very common adverse reaction) were monoclonal antibodies: adalimumab, alemtuzumab, atezolizumab, blinatumomab, brentuximab vedotin, cetuximab, ipilimumab, necitumumab, nivolumab, ocrelizumab, ofatumumab, palivizumab, panitumumab, pembrolizumab, pertuzumab, rituximab, siltuximab, trastuzumab, and trastuzumab emtansine. Thus, tyrosine kinase inhibitors and monoclonal antibodies represent together (slightly) over $40 \%$ of the medicines causing rash with high frequency.

Targeted and non-targeted anticancer medicinal products with different mechanisms of action are also well represented among the medicinal products frequently associated with rash. We identified 26 such products, besides the tyrosine kinase inhibitors or monoclonal antibodies shown above, representing $22.81 \%$ of the subclass: anastrozole, azacitidine, bicalutamide, busulfan, cladribine, cytarabine, docetaxel, doxorubicin, everolimus, fulvestrant, gemcitabine, hydroxycarbamide, idelalisib, ixazomib, lenalidomide, daunorubicin (liposomal), mitotane, paclitaxel, paclitaxel formulated as albumin bound nanoparticles, pemetrexed, pentostatin, raltitrexed, ribociclib, tamoxifen, temozolomide, temsirolimus, thiotepa and vismodegib.

A fourth group of medicinal products causing frequently rash consists of other biologicals: aldesleukin (IL-2), filgrastim, human immunoglobulin, interferon $\alpha-2 b$, interferon $\beta-1 b$, 
peginterferon $\alpha-2 b$, alglucosidase, asparaginase, idursulfase, laronidase, and sebelipase- $\alpha$.

A fifth group distinguished among the products for which rash is a very common adverse reaction is represented by various antivirals: efavirenz, etravirine, foscarnet, nevirapine, ribavirine, ritonavir, simeprevir, sofosbuvir, and tenofovir disoproxil.

A smaller, sixth group is represented by retinoids: acitretin, bexarotene, isotretinoin and tretinoin. Together, the six groups discussed above cover $84.21 \%$ of all medicines identified as causing rash with a relatively high frequency.

The remainder consists of various product with different indications and mechanisms of action: atovaquone, pyrimethamine (antiparasitics-antiprotozoal agents), atropine (anticholinergic alkaloid), carbamazepine, lamotrigine (antiepileptic/anticonvulsants), sirolimus (mTOR inhibitor, as everolimus and temsirolimus, already mentioned above among antitumor agents), glatiramer (polymer used in the treatment of multiple sclerosis), ivacaftor (CFTR potentiator used in the treatment of cystic fibrosis), pierfenidone (used in the treatment of idiopathic pulmonary fibrosis), terbinafine, voriconazole (antifungal), meptazinol (opioid analgesic), misoprostol (prostaglandin analog), strontium ranelate (used in the treatment of osteoporosis) and mesna (used to prevent urothelial toxicity in patients treated with ifosfamide and cyclophosphamide).

Rash as a 'common' adverse reaction. More than a quarter $(25.50 \%)$ of the medicinal products analysed had rash as a 'common' adverse reaction [Table SII (https://figshare.com/ articles/Untitled_Item/7144277)]. Monoclonal antibodies were also relatively well represented, numerically similar (18), but proportionally less $(\mathrm{P}=0.004)$ than among the group with rash as a 'very common' adverse reaction (6.74 vs. 16.67\%): avelumab, certolizumab pegol, daclizumab, denosumab, eculizumab, elotuzumab, evolocumab, golimumab, guselkumab, infliximab, inotuzumab ozogamicin, mepolizumab, natalizumab, obinutuzumab, ramucirumab, ranibizumab, tocilizumab, and vedolizumab. Unlike monoclonal antibodies, for which rash may be a 'very common' or just 'common', a single tyrosine kinase inhibitor was found to cause rash as a 'common' adverse reaction, tofacitinib.

The pharmacological or chemical classes of medicinal products having rash as a 'common' adverse event are more diverse than those having it as a 'very common' adverse reaction. The most frequent ones include:

a) Biologics (very diverse in their structure and indications, $12.36 \%$ of this frequency group): abatacept, belatacept, C1 inhibitor (human), darbepoetin alfa, epoetin alfa, epoetin zeta, follitropin $\beta$, goserelin, human plasma derived coagulation factor IX, icatibant acetate (peptidomimetic), imiglucerase, interferon $\beta$-1a, mifamurtide (small synthetic peptide), moroctocog, nonacog alfa, octreotide (and its acetate), peginterferon $\alpha-2 \mathrm{a}$, peginterferon- $\beta-1 \mathrm{a}$, pegvisomant, rabbit anti-human thymocyte immunoglobulin, romiplostim, simoctocog alfa, tasonermin [tumor necrosis factor- $\alpha$ (TNF- $\alpha)$ ], urofollitropin, agalsidase alfa, agalsidase- $\beta$, asfotase alfa, collagenase clostridium histolyticum, crisantaspase, rasburicase, streptokinase, and velaglucerase alfa.

b) Anticancer products, targeted and non-targeted $(10.11 \%$ of this frequency group): abiraterone, bleomycin, bortezomib, capecitabine, carboplatin, carfilzomib, clofarabine, decitabine, degarelix, epirubicin, eribulin, etoposide, exemestane, fludarabine, idarubicin, letrozole, mitomycin, niraparib, olaparib, oxaliplatin, panobinostat, pomalidomide, thalidomide, topotecan, toremifene, trabectedin, and vinflunine.

c) Antivirals (8.99\% of this frequency group): abacavir, aciclovir, adefovir, atazanavir, daclatasvir, darunavir, didanosine, dolutegravir, emtricitabine, famciclovir, fosamprenavir, ganciclovir, lamivudine, maraviroc, raltegravir, rilpivirine, saquinavir, stavudine, telbivudine, tenofovir alafenamide, tipranavir, valaciclovir, valganciclovir, and zanamivir.

d) Antibiotics and antituberculosis medications $(8.99 \%)$ : amoxicillin, avibactam, benzylpenicillin, cefaclor, cefadroxil, ceftaroline, ceftazidime, ceftobiprole, ceftriaxone, clarithromycin, daptomycin, delamanid, doxycycline, ertapenem, linezolid, meropenem, oritavancin, phenoxymethylpenicillin, rifabutin, rifaximin, teicoplanin, tigecycline, trimethoprim, and vancomycin.

e) Anticonvulsants (3.00\%): Eslicarbazepine, gabapentin, lacosamide, levetiracetam, oxcarbazepine, rufinamide, topiramate and zonisamide.

Monoclonal antibodies and these five additional classes represent half $(50.19 \%)$ of all products with rash as a 'common' adverse reaction. Other pharmacological classes, less represented among this frequency group include:

a) Antifungal agents: amphotericin B, anidulafungin, fluconazol, isavuconazole, itraconazole, micafungin and posaconazole.

b) Angiotensin-converting enzyme (ACE) inhibitors: captopril, enalapril, fosinopril, moexipril, perindopril arginine, perindopril erbumine and ramipril.

c) Antidepressants: clomipramine, duloxetine, fluoxetine, moclobemide, reboxetine, sertraline and venlafaxine.

d) Antipsychotic agents: haloperidol, lurasidone, olanzapine, olanzapine pamoate, paliperidone, risperidone and sulpiride.

e) Anticoagulants and platelet aggregation inhibitors: cilostazol, danaparoid, dipyridamole, edoxaban, enoxaparin, prasugrel, rivaroxaban and ticagrelor.

f) Agents interfering with opioid signaling: eluxadoline (mixed $\mu$-opioid receptor agonist and $\delta$-opioid receptor antagonist), morphine, naltrexone, oxycodone hydrochloride, racecadotril (enkephalinase inhibitor prodrug) and tapentadol.

g) NSAIDs: Acemetacin, celecoxib, diclofenac, nabumetone and piroxicam.

h) Ambrisentan, bosentan, epoprostenol, iloprost trometamol and selexipag.

i) Antidiabetic agents: Alogliptin, saxagliptin [dipeptidyl peptidase 4 (DPP-4) inhibitors], dapagliflozin, empagliflozin [sodium-glucose co-transporter 2 (SGLT2) inhibitors)].

j) Medicines used in the treatment of attention deficit hyperactivity disorder (ADHD): Atomoxetine, guanfacine, lisdexamfetamine dimesylate and methylphenidate.

k) Bone resorption inhibitors: Ibandronic acid, pamidronate, raloxifene and risedronate.

1) $\beta$-blockers: Acebutolol, celiprolol, labetalol and sotalol.

m) Diuretics: Chlortalidone, eplerenone and indapamide.

n) Lipid lowering medications: Acipimox, buprenorphine, ciprofibrate, gemfibrozil and lomitapide. 
o) Progestins: Medroxyprogesterone acetate, megestrol acetate and norethisterone enanthate.

A broad variety of other medicinal products also had rash as a 'common' adverse drug reaction: Acamprosate calcium (used in the treatment of alcohol dependence), lacidipine (the only calcium channel blocker), calcitriol, alfacalcidol (analogs/active forms of vitamin D), allopurinol, febuxostat (antigout medications), anagrelide (used for the reduction of elevated platelet counts), ataluren (for the treatment of Duchenne muscular dystrophy), atracurium, suxamethonium (neuromuscular blockers), baclofen, dantrolene (skeletal muscle relaxants), buspirone, meprobamate (anxiolytic), flumazenil (benzodiazepine antagonist), eprosartan (angiotensin receptor blocker), carbimazol (antithyroid), cimetidine $\left(\mathrm{H}_{2}\right.$ histamine receptor antagonist), cinacalcet (calcimimetic), cobicistat (pharmacokinetic enhancer), deferasirox (iron chelator), digoxin (cardiotonic), dimethyl fumarate, teriflunomide (medications for multiple sclerosis), dobutamine, midodrine (sympathomimetics), donepezil (cholinesterase inhibitor used in Alzheimer disease), memantine [N-methyl-D-aspartate (NMDA) blocker used in Alzheimer disease], rasagiline (antiparkinson), sodium oxybate (central nervous system depressant), dronedarone (antiarrhythmic), eltrombopag (thrombopoietin agonist), mesalazine, olsalazine (antiinflammatory agents for IBD), etomidate, ketamine (anaesthetics), hydroxychloroquine (antimalarial), pentamidine, tinidazole (antiprotozoal), lansoprazole (proton pump inhibitor), leflunomide [disease-modifying antirheumatic drugs (DMARDs)], montelukast, zafirlukast (leukotriene receptor antagonists), moxonidine (centrally acting anti-hypertensive), prazosin, terazosin ( $\alpha$-adrenoreceptor antagonists), mycophenolate mofetil, tacrolimus (immune suppressants), obeticholic acid (farnesoid $\mathrm{X}$ receptor agonist), mercaptamine (used in the cystinosis treatment), sodium phenylbutyrate (used in urea cycle disorders), pilocarpine (used orally in xerostomia), tadalafil [phosphodiesterase type 5 (PDE5) inhibitor], sugammadex (agent used for the reversal of neuromuscular blocking), tolvaptan (vasopressin receptor 2 antagonist), and varenicline ( $\alpha 4 \beta 2$ neuronal nicotinic acetylcholine receptor agonist).

Rash as an 'uncommon' adverse reaction. About one in every fifth (20.82\%) medicinal product used systemically has rash as an 'uncommon' adverse reaction [Table SIII (https:// figshare.com/articles/Untitled_Item/7144277)]. Only six monoclonal antibodies have associated rash with this lower frequency: Belimumab, ixekizumab, omalizumab, reslizumab, secukinumab and ustekinumab. No tyrosine kinase inhibitor has rash as an uncommon adverse reaction. The number of medicinal products used for the treatment of cancer and the number of antivirals are also very small, limited to five (chlorambucil, cisplatin, fluorouracil, irinotecan and pixantrone) and three molecules (entecavir, oseltamivir and zidovudine), respectively.

The pharmacological or chemical classes of medicinal products having rash as an 'uncommon' adverse event are as diverse as those with rash as a 'common' adverse reaction. The most frequent ones include:

a) Other biologics (than monoclonal antibodies), $11.47 \%$ among this frequency group: Aflibercept, albutrepenonacog alfa, anakinra, aprotinin, cetrorelix (synthetic peptide), conestat alfa, defibrotide, efmoroctocog, eptacog, etanercept, factor VIII, human $\alpha 1$-proteinase inhibitor, human fibrinogen, human insulin, insulin aspart, insulin detemir, insulin glulisine, mecasermin, octocog alfa, pegfilgrastim, reteplase, thyrotropin alfa, triptorelin acetate, triptorelin embonate and von Willebrand factor.

b) Antibiotics ( $8.26 \%$ among this frequency group): Amikacin, azithromycin, cefotaxime, cefpodoxime, cefuroxime, ciprofloxacin, clindamycin, dalbavancin, fidaxomicin, flucloxacillin, fosfomycin, levofloxacin, methenamine hippurate, moxifloxacin, ofloxacin, pivmecillinam, sodium fusidate and tedizolid.

c) NSAIDs (4.59\%): Aceclofenac, dexketoprofen, etoricoxib, flurbiprofen, ibuprofen, ibuprofen lysine, ketoprofen, meloxicam, parecoxib and tenoxicam.

d) Anticoagulants and platelet aggregation inhibitors (3.67\%): Apixaban, argatroban, aspirin, cangrelor, clopidogrel, dabigatran, fondaparinux and heparin.

e) Lipid lowering agents (3.21\%): Atorvastatin, bezafibrate, cholestyramine, ezetimibe, fenofibrate, pravastatin and rosuvastatin.

f) Anticonvulsants (3.21\%): Brivaracetam, fosphenytoin, pregabalin, primidone, retigabine, sodium valproate and vigabatrin.

g) Antidepressants (2.75\%): Amitriptyline, citalopram, escitalopram, fluvoxamine, nortriptyline and paroxetine.

h) Antiparkinson medications (2.75\%): Apomorphine, bromocriptine, cabergoline, pramipexole, procyclidine and selegiline.

i) Opioids (2.75\%): Dihydrocodeine, fentanyl citrate, hydromorphone, loperamide, sufentanil, tramadol and buprenorphine.

j) Antiemetics (2.75\%): Aprepitant, domperidone, fosaprepitant, granisetron, metoclopramide and palonosetron.

k) Antipsychotics (2.29\%): Asenapine, flupentixol, paliperidone palmitate, pimozide and quetiapine.

1) Antidiabetics (2.29\%): Canagliflozin (SGLT2 inhibitor), dulaglutide, liraglutide, lixisenatide [Glucagon-like peptide 1 (GLP-1) agonists] and linagliptin (DPP-4 inhibitor).

m) Various cardiovascular medications (13.30\%): Calcium channel blockers (1.83\%): Amlodipine, clevidipine, felodipine, nimodipine; angiotensin receptor blockers (1.83\%): Azilsartan, losartan, olmesartan, telmisartan; $\beta$-blockers (1.83\%): Carvedilol, metoprolol, nadolol, nebivolol; ACE inhibitors (1.83\%): Imidapril, lisinopril, quinapril, trandolapril; vasodilators and other antianginal medicines (2.75\%): Glyceryl trinitrate, isosorbid dinitrate, isosorbid mononitrate, naftidrofuryl oxalate, regadenoson, ivabradine); diuretics (1.83\%): Bumetanide, mannitol, spironolactone, triamterene; antiarrhythmics $(0.92 \%)$ : Flecainide, propafenone; antifibrinolytic: Tranexamic acid.

n) Proton pump inhibitors (1.83\%): Esomeprazole, omeprazole, pantoprazole and rabeprazole.

o) Anticholinergics (1.873): Darifenacin, fesoterodine, orphenadrine and tolterodine.

A broad variety of other medicinal products also had rash as an 'uncommon' adverse drug reaction: acetylcysteine, erdosteine (mucolytics); alendronate/alendronic acid, zoledronic acid (bone resorption inhibitors); plerixafor (immune stimulant used to augment mobilisation of 
haematopoietic stem cells to the peripheral blood for collection followed by transplantation); alfuzosin, doxazosin, tamsulosin ( $\alpha$-adrenoreceptor antagonists); mirabegron ( $\beta 3$-adrenoceptor agonist used in overactive bladder syndrome); progesterone, ulipristal acetate (progestins); testosterone undecanoate; macitentan (endothelin receptor antagonist); alitretinoin (retinoid); alprostadil, mifepristone (prostaglandins); apremilast, roflumilast (PDE4 inhibitors); sildenafil, vardenafil (PDE5 inhibitors); atosiban (oxytocin antagonist); azathioprine, ciclosporin, methotrexate (immunosuppresants); caspofungin (antifungals); cetirizine, levocetirizine, rupatadine (H1 histamine antagonists); cisatracurium, mivacurium (neuromuscular blockers); cyproterone (antiandrogen); deflazacort, triamcinolone acetonide (glucocorticoids); diazepam, midazolam maleate (benzodiazepines); eletriptan, rizatriptan (vascular 5- $\mathrm{HT}_{1 \mathrm{~B}}$ and neuronal 5- $\mathrm{HT}_{1 \mathrm{D}}$ agonists used as antimigraine medicines); ziconotide [N-type voltage-sensitive calcium channels (NCCB) inhibitor analgesic]; fampridine (potassium channel blocker used in multiple sclerosis); galantamine (cholinesterase inhibitor used in Alzheimer disease); pitolisant $\left(\mathrm{H}_{3}\right.$ histamine receptor inverse agonist used in the treatment of narcolepsy); riluzole (glutamate signaling inhibitor used in amyotrophic lateral sclerosis); glycerol phenylbutyrate (for urea cycle disorders); nitisinone (inhibitor of 4-hydroxyphenylpyruvate dioxygenase, used in hereditary tyrosinemia type 1); hexaminolevulinate, verteporfin (used in photodynamic therapy); paricalcitol (vitamin D analog); melatonin; nicotine, nicotine resinate; tocofersolan (PEG derivative of $\alpha$-tocopherol).

Rash as a 'rare' adverse reaction. Approximately $10 \%$ of the 1,048 medicinal product analysed are associated with rash as a 'rare' adverse event [Table SIV (https://figshare.com/articles/ Untitled_Item/7144277)]. A single monoclonal antibody belongs to this frequency group (abciximab) and no tyrosine kinase inhibitor. The chemical or pharmacological groups best represented among this frequency class $(\sim 60 \%)$ included:

a) Biologics (16.33\% among this frequency group): Alteplase, factor XIII, human albumin, human anti-D immunoglobulin, human hemin, human hepatitis B immunoglobulin, insulin degludec, insulin glargine, insulin lispro, interferon $\alpha-2 a$, methoxy polyethylene glycol-epoetin- $\beta$, oxytocin, somatropin, tenecteplase, teriparatide, and urokinase.

b) Anticancer medications (9.18\%): Bendamustine, cyclophosphamide, dacarbazine, flutamide, ifosfamide, melphalan, mercaptopurine, treosulfan and vinorelbine.

c) Benzodiazepines (6.12\%): Chlordiazepoxide, clonazepam, flurazepam, lorazepam, lormetazepam and nitrazepam.

d) Local anesthetics (4.08\%): Bupivacaine, chloroprocaine, prilocaine and ropivacaine.

e) $\beta$-blockers (4.08\%): Atenolol, bisoprolol, propranolol and timolol.

f) Vasodilators and antianginal (4.08\%): Hydralazine, minoxidil, nicorandil and ranolazine.

g) Anticoagulants (3.06\%): Acenocoumarol and bivalirudin.

h) Antiparkinson medications (3.06\%): Carbidopa, entacapone and safinamide.

i) Antiemetics (3.06\%): Droperidol, ondansetron, rolapitant.

j) Antibiotics and anti-tuberculosis medicines (3.06\%): ethambutol, minocycline and oxytetracycline. k) Vascular 5- $\mathrm{HT}_{1 \mathrm{~B}}$ and neuronal 5- $\mathrm{HT}_{1 \mathrm{D}}$ agonists (used as antimigraine treatments, 3.06\%): Frovatriptan, naratriptan and zolmitriptan.

1) Anticholinergics (3.06\%): Propiverine, solifenacin, trospium (used in overactive bladder syndrome).

Other medicinal products with rash as a 'rare' occurrence identified are: agomelatine (antidepressant), albiglutide (antidiabetic, GLP-1 agonist), nateglinide (antidiabetic, secretagogue which closes ATP-dependent potassium channels in the $\beta$-cell membrane), avanafil (PDE5 inhibitor), benperidol (antipsychotic), pizotifen (anti-serotonin and anti-tryptaminic used in migraine), phenytoin (anticonvulsant), rivastigmine (cholinesterase inhibitor used in Alzheimer), methocarbamol (central muscle relaxant), remifentanil (opioid analgesic), zopiclone (cyclopyrrolone hypnotic), bisacodyl (laxative), clemastine $\left(\mathrm{H}_{1}\right.$ histamine receptor antagonist), ranitidine ( $\mathrm{H}_{2}$ histamine receptor antagonist), desferrioxamine (iron chelating agents), desogestrel, drospirenone (progestins), estradiol, dexamfetamine (symphathomimetic), disodium clodronate (bone resorption inhibitor), lercanidipine, nifedipine (calcium channel blockers), fluvastatin, simvastatin (cholesterol lowering agents), furosemide, xipamide (diuretics), mebendazole (antiparasitic), prednisone (corticosteroid), sucralfate (gastric protective agent), Iomeprol (contrast medium), metyrapone (diagnostic agent for pituitary function), nicotine bitartrate, nicotine polacrilin, paracetamol, folic acid, hydroxocobalamin, and hydroxocobalamin acetate.

Rash as a 'very rare' adverse reaction. Only 3\% of the 1,048 medicines analysed had 'rash' listed as a 'very rare' event [Table SV (https://figshare.com/articles/Untitled_ Item/7144277)]. These are quite diverse with respect to their structure and pharmacological classification: A few biologics (choriogonadotropin alfa, follitropin alfa, ganirelix, glucagon, human coagulation factor VIII, lenograstim, lutropin alfa, protein C), antiarrhythmics (amiodarone, disopyramide), calcium channel blockers (verapamil), angiotensin receptor blockers (candesartan), cardiotonic agents (milrinone), platelet aggregation inhibitors (eptifibatide), diuretics (torasemide), benzodiazepines (clobazam), antipsychotics (clozapine), neuromuscular blockers (rocuronium, vecuronium), anaesthetics (propofol), antidiabetics agents (metformin), antiallergics (desloratadine, loratadine, mizolastine), antibiotics (gentamicin, metronidazole, metronidazole benzoate), progestins (levonorgestrel), bronchodilators (salbutamol), diagnostic agents (indigo carmin), ursodeoxycholic acid, folinic and levofolinic acid.

Medicines without rash as an adverse reaction. Within the 1,048 products included in the analysis $82(7.83 \%)$ did not listed 'rash' or an alternative term at all in the SmPC (Table SVI; https://figshare.com/articles/Untitled_Item/7144277). These were as diverse as the majority of groups, including:

a) Monoclonal antibodies (no less than 6: bezlotoxumab, brodalumab, canakinumab, daratumumab, dupilumab and idarucizumab).

b) Other biologics (beractant, follitropin- $\delta$, menotrophin, nusinersen, ocriplasmin, parathyroid hormone and somatorelin).

c) Two Janus kinase inhibitors (baricitinib and ruxolitinib).

d) One antiviral agent (dasabuvir). 
e) Four antitumor agents (lomustine, nelarabine, tioguanine and venetoclax).

f) Several opioid agonists and antagonists (alfentanil, methylnaltrexone, nalmefene and naloxone), as well as a synthetic cannabinoid (nabilone).

g) A number of products for which there is good reason to assume they are almost harmless: Adenosine, ascorbic acid, betaine, cholic acid, colesevelam, dihydrotachysterol, dopamine, ethanol, glycerol, lactulose, levocarnitine, levomenthol, magnesium aspartate, menadiol, methylcellulose, patiromer sorbitex (antidote for hyperkaliemia, is not absorbed or metabolized), pyridoxine, sodium citrate.

Besides the examples mentioned above, a broad variety of chemical structures and pharmacological groups belong to this class of products for which no rash is mentioned in the SmPCs: Antihistamines (bilastine), somatostatin analogs (pasireotide diaspartate and pamoate), an inhibitor of L-tryptophan hydroxylases, used in carcinoid syndrome diarrhoea (telotristat), a transthyretin stabilizer, used in familial amyloid polyneuropathy (tafamidis), a catecholamine-depleting medication used in hyperkinetic movement disorders (tetrabenazine), an androgen and anabolic steroid (nandrolone), a calcimimetic (etelcalcetide), a vassopresin analog (terlipressin), an oxytocin receptor agonist (carbetocin), a stimulator of soluble guanylate cyclase used in pulmonary hypertension (riociguat), a selective $\mathrm{D}_{2}$ receptor agonist used in hyperprolactinemia (quinagolide), a 5-HT4 receptor agonist used for constipation (prucalopride), a selective serotonine reuptake inhibitor used in premature ejaculation (dapoxetine), two inhibitors of glucosylceramide synthase used in Gaucher's disease (miglustat, eliglustat), two corticosteroids (beclometasone dipropionate, budesonide), two antiparkinson medications (opicapone, tolcapone), a reversible acetylcholinesterase inhibitor (edrophonium), an antiarrhythmic (vernakalant), several sympathomimetics (adrenaline, ephedrine, metaraminol), an $\alpha$-blocker (phenoxybenzamine), an antimuscarinic (propantheline), a sedative (dexmedetomidine), a benzodiazepine (alprazolam), an antipsychotic (amisulpride), an anticonvulsant (perampanel), a respiratory stimulant (doxapram), an antidote (dimercaprol), two diagnostic agents (tilmanocept, 5-aminolevulinic acid), anti-tuberculosis medication (bedaquiline) and two antiseptics (amylmetacresol, and hexylresorcinol).

Medicines for which rash frequency is not known. For 235 medicines of the 1,048 analysed (more often than one in every five), the frequency of rash as an adverse reaction is not estimated within the SmPCs [Table SVII (https://figshare.com/ articles/Untitled_Item/7144277)].

\section{Discussion}

It has been previously stated that almost all medicines have been associated with rash (7). Our analysis confirms that over $90 \%$ of the medicines used orally or by injection $(92.17 \%$ to be precise) may be associated with rash as an adverse event, but its frequency varies considerably. About one in every ten such products is associated very frequently with rash (more than $10 \%$ of the patients treated), and about one in every three medicines is associated very frequently or frequently with rash (more than $1 \%$ of the patients treated).
It is interesting to notice that the large majority of protein kinase inhibitors have rash as an adverse reaction with a very high frequency, except for tofacitinib (rash described as 'common'), baricitinib, and ruxolitinib (no rash at all). All three exceptions are inhibitors of Janus kinases, and it is interesting to contrast this minority with the high majority, for which rash is not only very frequent, but it may also be a useful marker for efficacy $(36,37)$. It is also interesting to note that whereas baricitinib has no rash as an adverse reaction, it does have acne as such a reaction, and the rash in the case of other protein kinase inhibitors may be acneiform (papulopustular) and be prevented by oral tetracyclines $(37,38)$. Although most protein kinase inhibitors do have rash as a very common adverse drug reaction, meta-analysis data indicate that there are differences among different molecules, e.g., it is significantly more frequent with afatinib $(84.8 \%)$ than with erlotinib $(62.0 \%)$ or gefitinib (62.0\%) (39). It seems that increased expression of IL-8, attracting neutrophils, is responsible for the occurrence of rash associated with these molecules, and its inhibition (e.g., with dapsone) is able to improve this adverse event (40). Our analysis was focused exclusively on the frequency of rash, irrespective of its severity or seriousness, since this information is available just in a small minority of SmPCs. Data from several tyrosine kinase inhibitors show that rash of grade 3/4 occurs with a much lower frequency, varying between 1 and $13 \%$, unlike all rashes, which have frequencies of $47 \%$ or higher (41).

The relationship between monoclonal antibodies and skin rash is at least interesting. Numerous monoclonal antibodies trigger the occurrence of rash with a high frequency. This should not necessarily be surprising, because they are foreign proteins and in a simplified scheme of things antibodies are produced to defend a host against foreign proteins (as parts of potential invaders) (42). More interesting, though, is the finding that although over 35 monoclonal antibodies have rash as a 'very common' or 'common' adverse reaction, there is a smaller number (seven in our analysis) of antibodies for which rash is an uncommon or rare event, and more interesting, there are monoclonal antibodies not associated with rash at all. Is there a scientific explanation for these differences? Is it related more to the target of these antibodies or to the production process, their chemical structure (primary structure, glycosylation patterns etc), or the expression system and purification steps applied in their production? The fact that bezlotoxumab is an antitoxin antibody, targeting a prokaryote protein and is associated with a very limited number of adverse events [its safety profile being similar to that of placebo (43)], whereas other antibodies targeting human proteins have a higher number of adverse events suggests that the safety profile is probably related more to the biological target than the expression system and purification of the antibody. However, the understanding of the impact of the different expression systems and glycosylation patterns on safety profiles is currently limited (44).

The majority of protein kinase inhibitors and many monoclonal antibodies are indicated in the treatment of cancer. Numerous other targeted and non-targeted anticancer therapies are also associated with rash with a moderate or high frequency, but rather curiously, there are also such therapies for which rash is an uncommon or rare event, and for a number of four molecules no rash was reported (lomustine, nelarabine, 
tioguanine and venetoclax). It is not clear what signalling pathways are involved in the rash associated with different anticancer therapies and why some of these otherwise relatively toxic medicines (e.g., lomustine or nelarabine) have (almost) no skin toxicity. The situation is similar for the increasingly large group of biologics: virtually in every frequency groups, biologics are present, suggesting that the fact that a molecule is a protein or otherwise a biological medicinal product, its administration does not necessarily associate with rash.

Most antivirals cause frequently rash, which among this therapeutic class is either very common or common. It is interesting, for instance, that whereas for tenofovir alafenamide the rash occurs as a 'common' adverse event, in the case of 'tenofovir disoproxil', according to the SmPCs, this adverse reaction is 'very common'. Although there is a perception of antibiotics (especially for penicillins) as having a high frequency of rash, they are causing generally less rash than antivirals, for most of them rash occurring as a 'common' or 'uncommon' adverse event. Many cardiovascular and neurological or psychiatric medications may also have rash as a 'common' or 'uncommon' adverse reaction, but few have rash as a 'very common' adverse reaction (a few anticonvulsants).

These assessments are impacted to some extent by the fact that for over $22 \%$ of the molecules analysed, the frequency of rash was not known in the approved SmPCs. In many cases this was stated explicitly (as frequency 'not known'), or implicitly, by not providing any information on rash frequency within the SmPC. It is likely that for most of these the frequency is moderate or low (e.g., rash should be 'uncommon', 'rare' or 'very rare'). In many cases, these adverse reactions have not been detected in clinical trials, but they have been identified in post-marketing surveillance. The fact that they did not occur in clinical trials suggests a low frequency, less than 1 in 100 or (more likely) still lower. Although this is not as essential as quantitative data on efficacy endpoints, we think that in the twentieth century more could be done to have at least a better estimate than 'not known', e.g., using meta-analyses of published clinical trials, using new clinical trials currently ongoing or using better methodologies of analysing spontaneous reporting data. Acetazolamide, for instance, according to the SmPC may cause various rashes, including erythema multiforme, Stevens-Johnson Syndrome and toxic epidermal necrolysis (45) and a limited number of case reports have been published (46-49). For these rashes, as well as for all other adverse drug reactions, no frequency information is available in the SmPCs. However, currently no less than 24 clinical trials with acetazolamide are recruiting or in preparation, according to the ClinicalTrials.gov database (50). It is a pity that in an era of increased pressure for transparency and openness on clinical trials, with hundreds of clinical trials published with acetazolamide (a PubMed query limited to clinical trials data this year returns over 500 publications), one is not able to perform an estimation of the frequency of an adverse event. This suggests that there is still a public need to include safety endpoints in clinical trials, even if the main endpoint is focused on efficacy, and to publish the results, as this would be in the interest of the society of large, as well as in the interest of the subjects included in the trials.

The number of SmPCs containing rash (and other adverse reactions) for which the frequency is not known (or no estimate is provided in the SmPC) is actually larger, because in many cases for the same molecule some SmPCs included a semi-quantitative estimate, whereas other(s) SmPC(s) did not; in such cases, however, we based our analysis on the SmPC including frequency data. It is disconcerting to know, though, that health professionals are provided information on the same molecule by chance, depending on the SmPC they happen to read; for a generic they may find that the frequency is not known, whereas for a different generic they are provided with a frequency estimation. In the case of carboplatin, for instance, there are two products available, both generics, in slightly different pharmaceutical forms (one is a solution for infusion, the other a concentrate for solution for infusion), but in the same concentration: for one, rash is stated as a 'common' adverse reaction (51), whereas for the other, the frequency is 'not known' (52). When an SmPC did not contain a tabular report on adverse events (including the CIOMS frequency classification), we have considered such cases as 'frequency not known', but the language used in such SmPCs may be confusing and contradicting the wording of those SmPC using the CIOMS frequency classification. For instance, whereas two digoxin SmPCs describe the rash as a 'common' occurrence $(53,54)$, a third one, not using the CIOMS frequency system, states that 'Hypersensitivity reactions have been reported rarely in patients taking digoxin'. These include pruritus, erythematous rashes, papules, vesicles and angioedema' (55). The contrast between 'common' and 'rarely' is sufficiently sharp to raise an eyebrow, although the frequency of rash is not the most important element of an SmPC.

Many such examples are available, but even more problematic are those SmPCs for the same active substance where different frequencies are provided for rash as an adverse reaction, all using the tabular reporting based on the CIOMS system. In certain cases, these may be due to different clinical data, generated in different conditions and with different dosages. For instance, dimethyl fumarate is authorized in UK by two different marketing authorization holders (MAHs), in two different indications (moderate to severe plaque psoriasis in adults and relapsing remitting multiple sclerosis), in different dosage forms (gastro-resistant tablets and capsules) and different strengths (30-120 and 120-240 mg, respectively). For the product authorized in relapsing remitting multiple sclerosis, rash is mentioned in the SmPC as a 'common' adverse reaction (56), whereas for the plaque psoriasis indication rash is not explicitly mentioned in the SmPC, but 'allergic skin reaction' is indicated as a 'rare' event, and this is based on data derived from 'Fumaderm, a related medicinal product containing dimethyl fumarate along with other fumaric acid esters' (57). In other cases, though, it is difficult to find a reasonable explanation for the differences seen in frequencies published in SmPCs, besides the use of old or partial data and lack of updating. In the case of doxorubicin (concentrates for) solutions for infusion/injection, for instance, whereas some SmPCs mention rash as a 'common' adverse event $(58,59)$, the SmPC of one generic concentrate for solution for infusion mentions 'exanthema' and 'urticaria' as 'rare' adverse events (60). In theory one could assume that the differences are derived from different formulations and clinical data, but in this case the product with a 'rare' rash is a generic, which by the regulatory standards do not come with 
its own data generated in full efficacy and safety trials, but through smaller bioequivalence studies. Moreover, we have looked into the public assessment report and have found that this specific generic has used the solution for injection as a European Reference Product in different EU member states, in the SmPC of which rash is mentioned as a 'common' adverse reaction (61).

There are also frequent ways of reporting 'rash' as an adverse event within the SmPCs. Not only are some SmPCs still in a narrative format, with no tabular content for adverse events and their frequencies (e.g., for edrophonium, ephedrine, gliclazide). For mebeverine, for instance, a very limited number of adverse events are included in section 4.8 of the SmPC, being stated that 'The following adverse reactions have been reported spontaneously during post-marketing use. A precise frequency cannot be estimated from available data' (62). However, more adverse events than those included in the SmPC are available from clinical trials, and could be used to update the SmPC. A mere PubMed search returned at least four easily accessible clinical trial publications (63-66), one in paediatric patients and three in adults (636 patients treated with mebeverine) in which safety data have been published; two additional trials available only as abstracts in PubMed reported no adverse event in $\sim 300$ additional patients treated with mebeverine $(67,68)$. More information needs to be retrieved on the $7 \%$ skin adverse reactions (coming from 20 patients) reported by Jones et al (65), but these data show that section 4.8 of the SmPC could as a matter of fact be updated with information on adverse event frequencies based on clinical data published. The European legislation (Directive 2001/83/EC) imposes an obligation on MAHs to 'forthwith supply to the competent authority any new information which may entail the amendment' of SmPC (69). It seems that whereas some MAHs have updated their SmPC with new information, others have failed to do so, and due to overload, regulatory authorities intervene reactively, rather than proactively, in the contents of SmPCs.

Finally, we have identified a broad variety of reporting rash in SmPCs, from one active substance to another, but also among different SmPCs for medicinal products containing the same active substance. For products containing atracurium, for instance, the same formulation and strength $(10 \mathrm{mg} / \mathrm{ml}$ solution for injection or infusion), one product states 'urticarial' only, as 'rare' (70), another states 'urticaria' only, as 'common' (71), and a third one lists 'rash' as 'common' and 'urticaria' as 'rare' (72). In many cases 'rash' is used with no qualifier, whereas in others, complex definitions are used, such as 'rash consists of one or more of the preferred terms of rash, drug eruption, rash macular, rash papular, erythema, rash maculo-papular, rash pruritic, and urticarial' (73). Despite the existence of a standardised medical terminology dictionary (MedDRA) whose use is compulsory for pharmaceutical companies, our research shows that there is still work to do in order to facilitate reporting of adverse events in a more uniform and standardized mode that should allow better comparisons across different products from different companies (taking into account the inherent limitations and constraints of such comparison exercises).

Further efforts are needed for increasing the homogeneity of rash frequency reporting in SmPCs, by using of CIOMS classification and MedDRA coding in a more standardized manner, for allowing informed and valid comparisons across different products from different companies.

\section{Acknowledgements}

Not applicable.

\section{Funding}

No funding was received.

\section{Availability of data and materials}

The datasets used and/or analyzed during the current study are available from the corresponding author on reasonable request.

\section{Authors' contributions}

RA conceived the study, collected the SmPCs, extracted and analysed computationally the data. MD checked the accuracy of the data, collected and extracted. RA, MD, FF and DB contributed equally to the design, analysis, interpretation of the data as well as to the writing and reviewing of the manuscript. All authors read and approved the final manuscript.

\section{Ethics approval and consent to participate}

No human or animal subjects were used for this study and no ethical approval or consent was needed.

\section{Patient consent for publication}

Not applicable.

\section{Competing interests}

RA has received consultancy and speakers' fees from various pharmaceutical companies. MD, FF and BD declare that they have no competing interests.

\section{References}

1. Hoeffel Rosa da I, Resner de Moraes M and Lovato B: Dermatology in Public Health Environments. In: A Comprehensive Textbook. Bonamigo RR and Dornelles SIT (eds). Springer, Switzerland, 2018.

2. Young JW and Shear NH: Cutaneous drug reactions in the elderly. Drugs Aging 34: 655-672, 2017.

3. Balakirski G and Merk HF: Cutaneous allergic drug reactions: Update on pathophysiology, diagnostic procedures and differential diagnosic. Cutan Ocul Toxicol 36: 307-316, 2017.

4. Chopra D, Sharma V, Kapoor R and Dwivedi S: An observational study of cutaneous adverse drug reactions in a teaching hospital. Int J Clin Pharm 37: 996-999, 2015.

5. Ding WY, Lee CK and Choon SE: Cutaneous adverse drug reactions seen in a tertiary hospital in Johor, Malaysia. Int J Dermatol 49: 834-841, 2010.

6. Nandha R, Gupta A and Hashmi A: Cutaneous adverse drug reactions in a tertiary care teaching hospital: A North Indian perspective. Int J Appl Basic Med Res 1: 50-53, 2011.

7. Wick JY: Drug-induced rash: Nuisance or threat? Consult Pharm 28: 160-166, 2013.

8. Redwood AJ, Pavlos RK, White KD and Phillips EJ: HLAs: Key regulators of T-cell-mediated drug hypersensitivity. HLA 91: 3-16, 2018. 
9. Demoly P, Adkinson NF, Brockow K, Castells M, Chiriac AM, Greenberger PA, Khan DA, Lang DM, Park HS, Pichler W, et al: International Consensus on drug allergy. Allergy 69: 420-437, 2014.

10. Gomes E, Cardoso MF, Praça F, Gomes L, Mariño E and Demoly P: Self-reported drug allergy in a general adult Portuguese population. Clin Exp Allergy 34: 1597-1601, 2004.

11. Jurado-Escobar R, Perkins JR, García-Martín E, Isidoro-García M, Doña I, Torres MJ and Cornejo-García JA: Update on the genetic basis of drug hypersensitivity reactions. J Investig Allergol Clin Immunol 27: 336-345, 2017.

12. Bircher AJ and Scherer Hofmeier K: Drug hypersensitivity reactions: Inconsistency in the use of the classification of immediate and nonimmediate reactions. J Allergy Clin Immunol 129: 263-266, 2012.

13. Chen CB, Abe R, Pan RY, Wang CW, Hung SI, Tsai YG and Chung WH: An updated review of the molecular mechanisms in drug hypersensitivity. J Immunol Res 2018: 6431694, 2018.

14. European Commission: A Guideline on summary of product characteristics (SmPC), 2009. https://ec.europa.eu/health/sites/ health/files/files/eudralex/vol-2/c/smpc guideline_rev2 en.pdf.

15. Arguello B, Salgado TM and Fernandez-Llimos F: Assessing the information in the Summaries of Product Characteristics for the use of medicines in pregnancy and lactation. Br J Clin Pharmacol 79: 537-544, 2015.

16. Vromans L, Doyle G, Petak-Opel S, Rödiger A, Röttgermann M, Schlüssel E and Stetter E: Shaping medicinal product information: A before and after study exploring physicians' perspectives on the summary of product characteristics. BMJ Open 3: e003033, 2013.

17. Arguello B and Fernandez-Llimos F: Clinical pharmacology information in summaries of product characteristics and package inserts. Clin Pharmacol Ther 82: 566-571, 2007.

18. Rougemont M, Ulrich S, Hiemke C, Corruble E and Baumann P. French summaries of product characteristics: Content in relation to therapeutic monitoring of psychotropic drugs. Fundam Clin Pharmacol 24: 377-384, 2010.

19. Ulrich S, Hiemke C, Laux G, Müller-Oerlinghausen B Havemann-Reinecke U, Riederer P, Zernig G and Baumann P, TDM group of the Arbeitsgemeinschaft Neuropsychopharmakologie und Pharmakopsychiatrie (AGNP): Value and actuality of the prescription information for therapeutic drug monitoring of psychopharmaceuticals: A comparison with the medico-scientific evidence. Pharmacopsychiatry 40: 121-127, 2007.

20. Salgado TM, Arguello B, Martinez-Martinez F, Benrimoj SI and Fernandez-Llimos F: Clinical relevance of information in the Summaries of Product Characteristics for dose adjustment in renal impairment. Eur J Clin Pharmacol 69: 1973-1979, 2013.

21. Salgado TM, Arguello B, Martinez-Martinez F, Benrimoj S and Fernandez-Llimos F: Lack of harmonisation in the classification of renal impairment in European Summaries of Product Characteristics. Intern Med J 45: 686-687, 2015.

22. Bergk V, Haefeli WE, Gasse C, Brenner H and Martin-Facklam M: Information deficits in the summary of product characteristics preclude an optimal management of drug interactions: A comparison with evidence from the literature. Eur J Clin Pharmacol 61: 327-335, 2005.

23. San Miguel MT, Martínez JA and Vargas E: Food-drug interactions in the summary of product characteristics of proprietary medicinal products. Eur J Clin Pharmacol 61: 77-83, 2005.

24. Hallersten A, Fürst W and Mezzasalma R: Physicians prefer greater detail in the biosimilar label (SmPC) - Results of a survey across seven European countries. Regul Toxicol Pharmacol 77: 275-281, 2016

25. Hoetzenecker W, Nägeli M, Mehra ET, Jensen AN, Saulite I, Schmid-Grendelmeier P, Guenova E, Cozzio A and French LE: Adverse cutaneous drug eruptions: Current understanding. Semin Immunopathol 38: 75-86, 2016.

26. Happel CS: Rash diagnostics: An update on the diagnosis of allergic rashes. Curr Opin Pediatr 29: 371-378, 2017.

27. National Clinical Guideline Centre: Drug Allergy: Diagnosis and Management of Drug Allergy in Adults, Children and Young People. https://www.ncbi.nlm.nih.gov/pubmed/25340226. Accessed May 20, 2019.

28. Bircher AJ: Drug hypersensitivity. In: Chemical Immunology and Allergy. Bergmann KC and Ring J (eds). Vol 100. Karger, Basel, pp120-131, 2014

29. Haasbach E, Hartmayer C, Hettler A, Sarnecka A, Wulle U, Ehrhardt C, Ludwig S and Planz O: Antiviral activity of Ladania067, an extract from wild black currant leaves against influenza A virus in vitro and in vivo. Front Microbiol 5: 171,2014.
30. Romano A, Valluzzi RL, Caruso C, Maggioletti M and Gaeta F: Non-immediate cutaneous reactions to beta-lactams: Approach to diagnosis. Curr Allergy Asthma Rep 17: 23, 2017.

31. Jones RT, Evans W, Mersfelder TL and Kavanaugh K: Rare Red Rashes: A case report of levetiracetam-induced cutaneous reaction and review of the literature. Am J Ther 23: e944-e946, 2016.

32. Bahrani E, Nunneley CE, Hsu S and Kass JS: Cutaneous adverse effects of neurologic medications. CNS Drugs 30: 245-267, 2016.

33. de Golian E, Kwong BY, Swetter SM and Pugliese SB: Cutaneous complications of targeted melanoma therapy. Curr Treat Options Oncol 17: 57, 2016.

34. Zulian F, Martini G, Gobber D, Plebani M, Zacchello F and Manners P: Triamcinolone acetonide and hexacetonide intra-articular treatment of symmetrical joints in juvenile idiopathic arthritis: A double-blind trial. Rheumatology (Oxford) 43: 128811291, 2004

35. R Development Core Team: R: A Language and Environment for Statistical Computing. R Foundation for Statistical Computing, Vienna, 2017. https://www.R-project.org. Accessed May 20, 2019.

36. Nasu S, Suzuki H, Shiroyama T, Tanaka A, Iwata K, Ryota N, Ueda Y, Takata SO, Masuhiro K, Morita S, et al: Skin rash can be a useful marker for afatinib efficacy. Anticancer Res 38: 1783-1788, 2018

37. Hichert V, Scholl C, Steffens M, Paul T, Schumann C, Rüdiger S, Boeck S, Heinemann V, Kächele V, Seufferlein T, et al: Predictive blood plasma biomarkers for EGFR inhibitor-induced skin rash. Oncotarget 8: 35193-35204, 2017.

38. van Doorn R and van Zuuren EJ: Epidermal growth factor receptor inhibitor-associated rash prevented by oral tetracyclines. Br J Dermatol 175: 1135-1136, 2016.

39. Ding PN, Lord SJ, Gebski V, Links M, Bray V, Gralla RJ, Yang JC and Lee CK: Risk of treatment-related toxicities from EGFR tyrosine kinase inhibitors: A meta-analysis of clinical trials of gefitinib, erlotinib, and afatinib in advanced EGFR-mutated non-small cell lung cancer. J Thorac Oncol 12: 633-643, 2017.

40. Boccellino M, Quagliuolo L, Alaia C, Grimaldi A, Addeo R, Nicoletti GF, Kast RE and Caraglia M: The strange connection between epidermal growth factor receptor tyrosine kinase inhibitors and dapsone: From rash mitigation to the increase in anti-tumor activity. Curr Med Res Opin 32: 1839-1848, 2016.

41. Tischer B,Huber R, Kraemer M and Lacouture ME: Dermatologic events from EGFR inhibitors: The issue of the missing patient voice. Support Care Cancer 25: 651-660, 2017.

42. Brown TA: Gene cloning and DNA analysis: an introduction. 6th edition. Wiley-Blackwell, Oxford, Hoboken, NJ, 2010.

43. Wilcox MH, Gerding DN, Poxton IR, Kelly C, Nathan R, Birch T, Cornely OA, Rahav G, Bouza E, Lee C, et al; MODIFY I and MODIFY II Investigators: Bezlotoxumab for prevention of recurrent clostridium difficile infection. N Engl J Med 376: 305-317, 2017.

44. Liu L: Antibody glycosylation and its impact on the pharmacokinetics and pharmacodynamics of monoclonal antibodies and Fc-fusion proteins. J Pharm Sci 104: 1866-1884, 2015.

45. Acetazolamide $250 \mathrm{mg}$ tablets. Summary of Product Characteristics, 2019. https://www.medicines.org.uk/emc/ product/2785/smpc. Accessed May 20, 2019.

46. Ogoshi M, Yamada Y and Tani M: Acute generalized exanthematic pustulosis induced by cefaclor and acetazolamide. Dermatology 184: 142-144, 1992 .

47. Spring M: Skin eruptions following the use of diamox. Ann Allergy 14: 41-43, 1956.

48. Ogasawara K, Tomitsuka N, Kobayashi M, Komoribayashi N, Fukuda T, Saitoh H, Inoue T and Ogawa A: Stevens-Johnson syndrome associated with intravenous acetazolamide administration for evaluation of cerebrovascular reactivity. Case report. Neurol Med Chir (Tokyo) 46: 161-163, 2006.

49. Her Y, Kil MS, Park JH, Kim CW and Kim SS: Stevens-Johnson syndrome induced by acetazolamide. J Dermatol 38: 272-275, 2011.

50. ClinicalTrials.gov: 24 Studies found for: Acetazolamide/ Recruiting, Not yet recruiting. Studies, 2018. https://clinicaltrials. gov/ct2/results? Accessed May 5, 2018.

51. Carboplatin $10 \mathrm{mg} / \mathrm{ml}$ concentrate for solution for infusion. Summary of Product Characteristics. 2012. https://www. medicines.org.uk/emc/product/6005/smpc. Accessed May 5, 2018.

52. Carboplatin $10 \mathrm{mg} / \mathrm{ml}$ Intravenous infusion. Summary of Product Characteristics, 2017. https://www.medicines.org.uk/ emc/product/3787/smpc. Accessed May 5, 2018. 
53. Digoxin 250 micrograms/ml Solution for injection. Summary of Product Characteristics, 2017. https://www.medicines.org.uk/ emc/product/5290/smpc. Accessed May 5, 2018.

54. Lanoxin 125 Tablets. Summary of Product Characteristics, 2018 https://www.medicines.org.uk/emc/product/5461. Accessed May 5, 2018.

55. Digoxin Tablets BP 125 micrograms. Summary of Product Characteristics, 2014. https://www.medicines.org.uk/emc/ product/5773/smpc. Accessed May 5, 2018.

56. Tecfidera $120 \mathrm{mg}$ and $240 \mathrm{mg}$ gastro-resistant hard capsules. Summary of Product Characteristics, 2018. https://www.medicines. org.uk/emc/product/5256. Accessed May 5, 2018.

57. Skilarence $120 \mathrm{mg}$ Gastro-resistant Tablets. Summary of Product Characteristics, 2017. https://www.medicines.org.uk/emc/ product $/ 7480$. Accessed May 5, 2018.

58. Doxorubicin Solution for injection. Summary of product characteristics, 2016. https://www.medicines.org.uk/emc/product/6184. Accessed May 5, 2018.

59. Doxorubicin (Myocet) $50 \mathrm{mg}$ powder, dispersion and solvent for concentrate for dispersion for infusion. Summary of Product Characteristics, 2015. https://www.medicines.org.uk/emc/ product/5378. Accessed May 5, 2018.

60. Doxorubicin $2 \mathrm{mg} / \mathrm{ml}$ Concentrate for Solution for infusion. Summary of Product Characteristics, 2016. https://www. medicines.org.uk/emc/product/6112/smpc. Accessed May 5, 2018.

61. MHRA: Public Assessment Report. Decentralized procedure. Doxorubicin $2 \mathrm{mg} / \mathrm{ml}$ Concentrate for Solution for infusion (doxorubicin hydrochloride). UK/H/1347/001/DC. UK Licence numbers: PL20075/0109, 2010. https://www.medicines.org.uk/ emc/product/8443/smpc. Accessed May 5, 2018.

62. Aurobeverine MR $200 \mathrm{mg}$ modified-release capsules, hard. Summary of Product Characteristics, 2017. https://www. medicines.org.uk/emc/product/8994. Accessed May 5, 2018.

63. Pourmoghaddas Z, Saneian H, Roohafza H and Gholamrezaei A: Mebeverine for pediatric functional abdominal pain: A randomized, placebo-controlled trial. BioMed Res Int 2014: $191026,2014$.

64. Lee KJ, Kim NY, Kwon JK, Huh KC, Lee OY, Lee JS, Choi SC, Sohn CI, Myung SJ, Park HJ, et al: Efficacy of ramosetron in the treatment of male patients with irritable bowel syndrome with diarrhea: A multicenter, randomized clinical trial, compared with mebeverine. Neurogastroenterol Motil 23: 1098-1104, 2011.
65. Jones RH,Holtmann G, Rodrigo L, Ehsanullah RS, Crompton PM, Jacques LA and Mills JG: Alosetron relieves pain and improves bowel function compared with mebeverine in female nonconstipated irritable bowel syndrome patients. Aliment Pharmacol Ther 13: 1419-1427, 1999.

66. Van Outryve M, Mayeur S, Meeus MA, Rosillon D, Hendrickx B and Ceuppens M: A double-blind crossover comparison study of the safety and efficacy of mebeverine with mebeverine sustained release in the treatment of irritable bowel syndrome. J Clin Pharm Ther 20: 277-282, 1995.

67. Rahman MZ, Ahmed DS, Mahmuduzzaman M, Rahman MA, Chowdhury MS, Barua R and Ishaque SM: Comparative efficacy and safety of trimebutine versus mebeverine in the treatment of irritable bowel syndrome. Mymensingh Med J 23: 105-113, 2014

68. Gilbody JS, Fletcher CP, Hughes IW and Kidman SP: Comparison of two different formulations of mebeverine hydrochloride in irritable bowel syndrome. Int J Clin Pract 54: 461-464, 2000.

69. Directive 2001/83/EC of the European Parliament and of the Council of 6 November 2001 on the Community Code relating to Medicinal Products for Human Use, as amended. Official Journal L 311, pp67-128, 2004. Accessed May 5, 2018.

70. Atracurium $10 \mathrm{mg} / \mathrm{ml}$ Solution for injection or infusion. Summary of Product Characteristics, 2014. https://www.medicines.org.uk/ emc/search. Accessed May 5, 2018.

71. Atracurium $10 \mathrm{mg} / \mathrm{ml}$ Solution for injection/infusion. Summary of Product Characteristics, 2013. https://www.medicines.org.uk/ emc/product/5729. Accessed May 5, 2018.

72. Atracurium Besilate $10 \mathrm{mg} / \mathrm{ml}$ Solution for injection - ampoule. Summary of Product Characteristics, 2017. https://www. medicines.org.uk/emc/search. Accessed May 5, 2018.

73. Aptivus $100 \mathrm{mg} / \mathrm{ml}$ oral solution. Summary of Product Characteristics, 2017. https://www.medicines.org.uk/emc/ product/4451. Accessed May 5, 2018.

This work is licensed under a Creative Commons Attribution-NonCommercial-NoDerivatives 4.0 International (CC BY-NC-ND 4.0) License. 